\title{
TIDAL TORQUES DYNAMICAL FRICTION AND THE STRUCTURE OF CLUSTERS OF GALAXIES
}

\author{
A. DEL POPOLO, AND M. GAMBERA \\ Istituto di Astronomia Viale A.Doria, 6 - I 95125 Catania, \\ ITALY
}

\begin{abstract}
We study the joint effect of tidal torques and dynamical friction on the collapse of density peaks solving numerically the equations of motion of a shell of barionic matter falling into the central regions of a cluster of galaxies. We calculate the evolution of the expansion parameter, $a(t)$, of the perturbation using a coefficient of dynamical friction $\eta_{c l}$ obtained from a clustered system and taking into account the gravitational interaction of the quadrupole moment of the system with the tidal field of the matter of the neighboring proto-galaxies. We find that the tidal torques and the dynamical friction slow down the collapse of low- $\nu(\nu<3)$ peaks producing an observable variation of $a(t)$ (Del Popolo \& Gambera 1996,1997). As consequence we have a reduction of the mass bound to collapsed perturbations and a raising of the critical threshold, $\delta_{c}$. Besides, we have a bias of dynamical nature arises because high-density peaks preferentially collapse to form halos within which visible objects. We calculate the selection function and using it and the prescriptions given by Bardeen et al. 1986 we find a value of the coefficient of bias, $b=2.25$ on clusters scales for $R_{f}=4 h^{-1} M p c$ comparable both with that obtained from the mean mass-to-light ratio of clusters, APM survey, or from N-body simulations combined with hydrodynamical models and with the values of $b$ given by Kauffmann et al. 1996. This means that non-radial motions and dynamical friction play a significant role in determining the bias level.
\end{abstract}

\section{References}

Bardeen, J. M., Bond, J. R., Kaiser, N., Szalay, A. S. 1986, ApJ., 304, 15

Del Popolo, A., Gambera, M. 1996, A \& A 308, 373

Del Popolo, A., Gambera, M. 1997, A \& A 321, 691

Kauffmann, G., Nusser, A., Steinmetz, M., 1996, submitted to MNRAS 